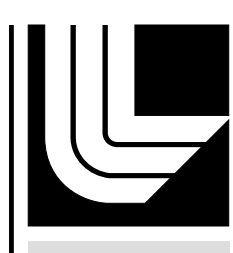

LAWRENCE LIVERMORE N A TIO NAL LABORATORY

\title{
Transient evolution of a photon gas in the nonlinear QED vacuum
}

S. S. Q. Wu, F. V. Hartemann

October 5, 2011 
This document was prepared as an account of work sponsored by an agency of the United States government. Neither the United States government nor Lawrence Livermore National Security, LLC, nor any of their employees makes any warranty, expressed or implied, or assumes any legal liability or responsibility for the accuracy, completeness, or usefulness of any information, apparatus, product, or process disclosed, or represents that its use would not infringe privately owned rights. Reference herein to any specific commercial product, process, or service by trade name, trademark, manufacturer, or otherwise does not necessarily constitute or imply its endorsement, recommendation, or favoring by the United States government or Lawrence Livermore National Security, LLC. The views and opinions of authors expressed herein do not necessarily state or reflect those of the United States government or Lawrence Livermore National Security, LLC, and shall not be used for advertising or product endorsement purposes.

This work performed under the auspices of the U.S. Department of Energy by Lawrence Livermore National Laboratory under Contract DE-AC52-07NA27344. 


\title{
Transient evolution of a photon gas in the nonlinear QED vacuum
}

\author{
S.S.Q. Wu and F.V. Hartemann
}

Lawrence Livermore National Laboratory, Livermore, CA 94550

\begin{abstract}
Thermally induced vacuum polarization stemming from QED radiative corrections to the electromagnetic field equations is studied. The physical behavior of thermal radiation, in the nonlinear QED vacuum first described by Heisenberg and Euler, is a problem of some theoretical importance in view of its relation to the cosmic microwave background (CMB), early universe evolution, and Hawking-Unruh radiation. The questions of evolution toward equilibrium, stability, and invariance of thermal radiation under such conditions are of great interest. Our analysis presents novel aspects associated with photon-photon scattering in a photon gas in the framework of quantum kinetic theory. Within the context of the Euler-Heisenberg theory, we show that a homogeneous, isotropic photon gas with arbitrary spectral distribution function evolves toward an equilibrium state with a Bose-Einstein distribution. The transient evolution toward equilibrium of a gas of photons undergoing photon-photon scattering is studied in detail via the Boltzmann transport equation.
\end{abstract}


The physical behavior of thermal radiation, in the nonlinear QED vacuum first described by Heisenberg and Euler [1], is a problem of some theoretical importance [2] in view of its relation to the cosmic microwave background (CMB) [3], early universe evolution [4], and HawkingUnruh radiation [5]. In particular, the questions of the evolution toward equilibrium, stability, and invariance of thermal radiation under such conditions are of great interest. In QED, the scattering of light by light is described as the result of the production of a virtual electronposition pair by the two initial photons, followed by the annihilation of the pair into the final photons. The probability of this process to occur, or equivalently, the scattering cross section, is calculated by summing contributions from each of the six "square" Feynman diagrams which describe the interaction [6]. In this paper, this problem is addressed within the context of the photon-photon scattering cross-section and it will be shown that, under specific assumptions defined below, a photon gas with an arbitrary spectral distribution function evolves toward an equilibrium state with a Bose-Einstein distribution. The path followed here describes interactions in terms of a differential scattering cross-section, which is directly related to the Smatrix formalism of QED and manifestly conserves all important physical parameters, including energy, momentum, spin, and charge. The transient evolution of a gas of photons in the presence of photon-photon scattering is described by the Boltzmann transport equation. The fundamental description attained here is thus relatively simple, intuitive and transparent.

Let us first recall some general characteristics of a gas of particles undergoing elastic scattering. It is well known from kinetic theory that a statistical ensemble of interacting particles in a nonequilibrium macrostate left to itself will tend toward a state of equilibrium in accordance with the Boltzmann transport equation,

$$
\frac{d n_{1}}{d t}=\int R\left(\mathbf{p}_{1}, \mathbf{p}_{2} ; \mathbf{p}_{1}^{\prime}, \mathbf{p}_{2}^{\prime}\right)\left[n_{1}^{\prime} n_{2}^{\prime}\left(1+\chi n_{1}\right)\left(1+\chi n_{2}\right)-n_{1} n_{2}\left(1+\chi n_{1}^{\prime}\right)\left(1+\chi n_{2}^{\prime}\right)\right] \frac{d^{3} \mathbf{p}_{1}^{\prime}}{(2 \pi)^{3}} \frac{d^{3} \mathbf{p}_{2}^{\prime}}{(2 \pi)^{3}} \frac{d^{3} \mathbf{p}_{2}}{(2 \pi)^{3}}(1)
$$

where $n_{1}, n_{2}, n_{1}^{\prime}, n_{2}^{\prime}$ are distribution functions of the momenta $\mathbf{p}_{1}, \mathbf{p}_{2} ; \mathbf{p}_{1}^{\prime}, \mathbf{p}_{2}^{\prime}$ of the colliding particles and $R$ is the collision kernel containing the detailed physics of the specific scattering process $[7,8,9]$. The two terms in the square brackets give respectively the numbers of particles entering and leaving a particular state as a result of collisions. The parameter $\chi$, which may 
take on three different values, $\chi=\{-1,0,1\}$, allows for the study of fermions and bosons. For elastic two-particle collisions, there are five independent collision invariants; namely, the total energy $E$, the three components of momentum and the particle number. One can verify that these quantities are conserved by equation (1). According to Boltzmann's H theorem, this process must be accompanied by an increase in the entropy of the gas until equilibrium is reached when the entropy production ceases. The condition of vanishing entropy production determines the equilibrium distribution function, given by:

$$
n=\frac{1}{\exp (\alpha+\beta E+\mathbf{U} \cdot \mathbf{p})-\chi}
$$

Indeed, the integral in equation (1) vanishes identically for distribution functions of this form. These are the familiar Maxwell-Boltzmann $(\chi=0)$, Fermi-Dirac $(\chi=-1)$ and Bose-Einstein ( $\chi=1$ ) distribution functions in a coordinate frame with bulk velocity $\mathbf{U}$. In the rest frame of the gas, $\mathbf{U}=0$, the distribution functions are characterized by the constants $\alpha$ and $\beta$ determined by number density and energy density conservation. It is worth noting that the formalism described above is very general and can be applied to a broad class of scattering problems, including collisions in plasma, quasi-particles in a Fermi liquid, and the Comptonization of isotropic photons by electrons. Comptonization of the $\mathrm{CMB}$, indeed, has been an area of interest in the astrophysical arena for many years [10]. In the Thomson limit, one typically approximates equation (1) by invoking the Fokker-Planck expansion and obtains a differential equation first described by Kompaneets [11]. The Kompaneets equation then describes the time evolution of photons and electrons toward their respective equilibrium distributions given by equation (2). In this paper, we will apply this general approach to derive the time evolution of an isolated photon gas undergoing nonlinear photon-photon scattering. It is evident before diving into specifics of the collision kernel that the equilibrium distribution will follow Bose-Einstein statistics for the reasons discussed above. It is, however, of interest to know how an arbitrary photon gas tends to its final equilibrium state, i.e. the transient evolution of the photon distribution function in the presence of photon-photon scattering. 
The technical steps of the derivation start from the photon-photon differential scattering rate. The total scattering rate is expressed in terms of manifestly covariant quantities in the S-matrix formulation. Next, using the symmetries of the problem, the differential rates of production of scattered photons and loss of incident photons are derived for arbitrary isotropic spectral distributions. Finally, the integro-differential evolution equation thus obtained is applied to specific cases of interest. Explicit calculations are carried out showing transient evolution of a photon gas under photon-photon scattering.

It is also important to emphasize the assumptions under which the derivation is performed and our conclusions are valid. First and foremost, the Heisenberg-Euler theory must hold - the photon energy is small compared to the electron rest energy and fields weak relative to the Schwinger critical field. Second, the mean free path between photon-photon collisions must remain much longer than the wavelengths involved, so that the photon energy is well defined. Another key point is that the photon number density and energy density conservation are conserved. Interaction with matter can change one or both quantities, hence, for this assumption to be valid, there must be negligible matter content in the region of interest. While 4-momentum conservation and photon number conservation are evident requirements for a given interaction, volumetric density conservation implies that the system is homogeneous, isotropic and unbounded, and that the interaction time scale is short compared to all other time scales. In reality, at any given time, part of the energy-momentum content of the system appears in the form of electron-positron pairs, thus diminishing the electromagnetic component of the 4momentum and introducing more scattering mechanisms; this is neglected in the present analysis.

The transition rate density $d W$ of a general scattering process in which two incident particles collide and are transformed into two outgoing particles, $h+k \rightarrow h^{\prime}+k^{\prime}$, is [6]:

$$
d W=\frac{1}{4 E_{h} E_{k}}|M|^{2}(2 \pi)^{4} \delta^{4}\left(p_{h}{ }^{\mu}+p_{k}{ }^{\mu}-p_{h}{ }^{\mu^{\prime}}-p_{k}{ }^{\mu^{\prime}}\right) \frac{d^{3} \mathbf{p}_{h}{ }^{\prime}}{(2 \pi)^{3} 2 E_{h}{ }^{\prime}} \frac{d^{3} \mathbf{p}_{k}{ }^{\prime}}{(2 \pi)^{3} 2 E_{k}{ }^{\prime}} .
$$


Natural units are used throughout: $\hbar=c=1$. Energy is measured with respect to the electron rest energy. For photons, $p_{k}{ }^{\mu}=\left(E_{k}, \mathbf{p}_{k}\right)=(k, \mathbf{k})=k^{\mu}$ and $k^{\mu} k_{\mu}=0$. Photon-photon scattering in vacuum is a well known quantum electrodynamic process. The magnitude square of the scattering amplitude $|M|^{2}$ for unpolarized photon-photon scattering in the Euler-Heisenberg regime of low energies $(k \ll m)$ expressed in terms of the Mandelstam variables [6],

$$
\begin{gathered}
s=\left(h^{\mu}+k^{\mu}\right)^{2}, t=\left(k^{\mu}-k^{\mu^{\prime}}\right)^{2}, u=\left(h^{\mu}-k^{\mu^{\prime}}\right)^{2} \text { with } s+t+u=0, \text { is: } \\
|M|^{2}=\frac{139 e^{8}}{2025 m^{8}}\left(t^{2}+t u+u^{2}\right)^{2} .
\end{gathered}
$$

In the center of mass (CM) frame where the process is most often presented, both incident and scattered photons have the same energy, $h=k=h^{\prime}=k^{\prime}$, as illustrated in Figure 1, and for energies much less than the electron mass, the photon-photon differential scattering cross-section is given by [6]:

$$
\frac{d \sigma}{d \Omega}=\frac{139 e^{8}}{4 \pi^{2} 90^{2} m^{8}} k_{C M}{ }^{6}\left(3+\cos ^{2} \theta_{C M}\right)^{2}
$$

where $k_{C M}$ and $\theta_{C M}$ are quantities measured in the CM frame.

Continuing with the transition rate density calculation, the three-dimensional delta function in momentum space can be eliminated by integration over $d^{3} \mathbf{p}_{h}{ }^{\prime}$ and noting $\mathbf{p}_{h}{ }^{\prime}=\mathbf{p}_{h}+\mathbf{p}_{k}-\mathbf{p}_{k}{ }^{\prime}$. Here it will be convenient to apply the remaining energy conserving delta function to an angular integral rather than a radial integral as is customary. Using the relation [12],

$$
\begin{aligned}
\int \frac{d^{3} \mathbf{p}_{h}{ }^{\prime}}{2 E_{h}{ }^{\prime}} \delta^{4}\left(p_{h}{ }^{\mu}+p_{k}{ }^{\mu}-p_{h}{ }^{\mu^{\prime}}-p_{k}{ }^{\mu^{\prime}}\right) & =\delta\left[\left(p_{h}{ }^{\mu}+p_{k}{ }^{\mu}-p_{k}{ }^{\mu^{\prime}}\right)^{2}-p_{h}{ }^{\mu^{\prime}} p_{h \mu}{ }^{\prime}\right] \\
& =\delta\left[2 h^{\mu} k_{\mu}-2\left(h^{\mu}+k^{\mu}\right) k_{\mu}{ }^{\prime}\right]
\end{aligned}
$$


and applying the same analysis to the time reversed process, equation (1) becomes

$$
\left(\frac{d n_{k}^{\prime}}{d t}\right)_{\text {collision }}=\frac{1}{(2 \pi)^{3}} \int W\left(\mathbf{h}, \mathbf{k} ; \mathbf{h}^{\prime}, \mathbf{k}^{\prime}\right)\left[n_{h} n_{k}\left(1+n_{h}{ }^{\prime}\right)\left(1+n_{k}{ }^{\prime}\right)-n_{h}^{\prime} n_{k}^{\prime}\left(1+n_{h}\right)\left(1+n_{k}\right)\right] d^{3} \mathbf{h} d^{3} \mathbf{k}
$$

where,

$$
W\left(\mathbf{h}, \mathbf{k} ; \mathbf{h}^{\prime}, \mathbf{k}^{\prime}\right)=\frac{1}{(2 \pi)^{2}} \frac{139 e^{8}}{2025 m^{8}} \frac{1}{8 h k k^{\prime}}\left(t^{2}+t u+u^{2}\right)^{2} \delta(s+t+u)
$$

The incident 4-wavenumbers defined in spherical coordinates are:

$$
\begin{aligned}
& h^{\mu}=h(1, \sin \theta \cos \phi, \sin \theta \sin \phi, \cos \theta) \\
& k^{\mu}=k(1, \sin \psi \cos \varphi, \sin \psi \sin \varphi, \cos \psi) .
\end{aligned}
$$

Note that since the problem is isotropic by construction, we may align the z-axis with the scattered photon, $k^{\mu^{\prime}}=q(1,0,0,1)$, without loss of generality. The solid angle differential elements are $d \Omega_{h}=\sin \theta d \theta d \phi$ and $d \Omega_{k}=\sin \psi d \psi d \varphi$. The Mandelstam variables now read:

$$
\begin{aligned}
& s=2 h^{\mu} k_{\mu}=2 h k[1-\cos \theta \cos \psi-\cos (\phi-\varphi) \sin \theta \sin \psi] \\
& t=-2 k^{\mu} k_{\mu}{ }^{\prime}=-2 k q(1-\cos \theta) \\
& u=-2 h^{\mu} k_{\mu}{ }^{\prime}=-2 h q(1-\cos \psi) .
\end{aligned}
$$

As expected from symmetry considerations, the azimuthal angles only appear as a difference, $\Delta=\phi-\varphi$, so we may write $\iint d \phi d \varphi \rightarrow 2 \pi \int d \Delta$. The integral over the relative azimuthal angle $\Delta$ is readily performed using the well-known sifting property of the delta-function: $\delta[g(x)]=\sum_{i} \delta\left(x-x_{i}\right) /\left|g^{\prime}\left(x_{i}\right)\right|$, where the $x_{i}$ are the zeroes of $g$. Here, the two zeroes of $s+t+u$ are located at: 


$$
\cos \Delta_{ \pm}=\frac{k q(x-1)+h q(y-1)+h k(1-x y)}{h k \sqrt{\left(x^{2}-1\right)\left(y^{2}-1\right)}}
$$

after a change of variables, $x=\cos \theta$ and $y=\cos \psi$. Proceeding with the integration over $\Delta$, let us define a function $f(h, k, q)$ where

$$
\begin{aligned}
f(h, k, q) & \equiv 2 \pi \int_{-1}^{1} d x \int_{-1}^{1} d y \int_{0}^{2 \pi} \frac{1}{(2 \pi)^{2}} \frac{1}{8 h k q}\left(t^{2}+t u+u^{2}\right)^{2} \delta(s+t+u) d \Delta \\
& =\frac{q^{3}}{\pi h^{2} k^{2}} \int_{-1}^{1} d x \int_{-1}^{1} d y \frac{\left[k^{2}(1-x)^{2}+h k(1-x)(1-y)+h^{2}(1-y)^{2}\right]^{2}}{\sqrt{\left(1-x^{2}\right)\left(1-y^{2}\right)\left(1-\cos ^{2} \Delta_{ \pm}\right)}} \Theta\left(1-\cos ^{2} \Delta_{ \pm}\right) .
\end{aligned}
$$

The Heaviside $\Theta$-function arises from the requirement $0 \leq \Delta_{ \pm} \leq 2 \pi$ and ensures that the integrand is real. To proceed, we note that the numerator in equation (12) is a simple polynomial over $x$ and $y$; in addition, the argument inside the square root in the denominator can be expressed in terms of its roots, $x_{ \pm}$:

$$
x_{ \pm}=\frac{k(h y-q)[k(h-q)+h q(y-1)] \pm \sqrt{h^{3} k^{2} q(y-1)^{2}(y+1)\left[2 k(h-q)+h q(y-1)+2 k^{2}\right]}}{k^{2}\left(h^{2}-2 h q y+q^{2}\right)}
$$

Therefore, the integral over $x$ can be rewritten in the form [13]:

$$
I_{n}=\int_{x_{-}}^{x_{+}} \frac{x^{n}}{\sqrt{\left(x-x_{+}\right)\left(x-x_{-}\right)}} d x=-i \pi\left(x_{-}\right)^{n}{ }_{2} \mathrm{~F}_{1}\left(\frac{1}{2},-n ; 1 ; 1-\frac{x_{+}}{x_{-}}\right)
$$

Here, ${ }_{2} \mathrm{~F}_{1}(a, b ; c ; x)$ is the Gauss hypergeometric function. Values of $n$ range from 0 to 4 . We may rewrite $f$ as

$$
f(h, k, q)=\int_{-1}^{1} d y \sum_{n=0}^{4} \frac{a_{n}(h, k, q, y)}{\left(h^{2}-2 h q y+q^{2}\right)^{n+\frac{1}{2}}}
$$


where $a_{n}$ are polynomials in $y$.

By inspecting equation (12), it is clear that special attention must be paid to the boundaries of the integration domain. The $y$ integral in equation (15) can be approached by first dividing it into two disparate cases. When $q<k$, the integration region covers the entire interval $y \in[-1,1]$; when $q>k$, however, the integration region shrinks to $y \in\left[y_{1}, 1\right]$, where the lower bound is $y_{1}=1+\frac{2 k}{h}-\frac{2 k}{q}-\frac{2 k^{2}}{h q}$. Integration over $y$ can now be performed in terms of the incomplete beta function, $\mathrm{B}(x ; a, b)[13]$ :

$$
\int_{y_{1}}^{1} \frac{y^{m}}{\left(y_{0}-y\right)^{n+\frac{1}{2}}} d y=y_{0}^{\frac{1}{2}+m-n}\left[\mathrm{~B}\left(\frac{1}{y_{0}} ; 1+m, \frac{1}{2}-n\right)-\mathrm{B}\left(\frac{y_{1}}{y_{0}} ; 1+m, \frac{1}{2}-n\right)\right]
$$

with $y_{0}=\frac{1}{2}\left(\frac{h}{q}+\frac{q}{h}\right)$.

For $q<k$, after integration over $y$, the function $f$ itself can be further subdivided into two regions based on the value of $h$ :

$$
\begin{aligned}
& f_{I}=\frac{32 q^{3}}{35 h^{2} k^{2}}\left(2 q^{2}\left(8 h^{2}+15 h k+8 k^{2}\right)-7 q(h+k)\left(2 h^{2}+3 h k+2 k^{2}\right)+7\left(h^{2}+h k+k^{2}\right)^{2}-9 q^{3}(h+k)+2 q^{4}\right), \begin{array}{c}
q<h \\
q<k
\end{array} \\
& f_{I I}=\frac{32 h^{3}}{35 k^{2} q^{2}}\left(2 h^{4}+9 h^{3} k+q^{2}\left(16 h^{2}+35 h k+21 k^{2}\right)-q(h+k)\left(9 h^{2}+21 h k+14 k^{2}\right)+16 h^{2} k^{2}+14 h k^{3}-14 q^{3}(h+k)+7 k^{4}+7 q^{4}\right), \quad h<q<k
\end{aligned}
$$

This second division is a reflection of symmetry in the problem. Other than the order of integration, neither $h$ nor $k$ is special; hence any feature in the domain of one variable must be reflected in the domain of the other.

Similarly, for the case when $q>k$, the expression after integration over $y$ divides into two subdomains: 


$$
\begin{aligned}
& f_{I I I}=\frac{32 k^{3}}{35 h^{2} q^{2}}\left(2 k^{2}\left(8 h^{2}-15 h q+8 q^{2}\right)+7 k(h-q)\left(2 h^{2}-3 h q+2 q^{2}\right)+7\left(h^{2}-h q+q^{2}\right)^{2}+9 k^{3}(h-q)+2 k^{4}\right), \quad k<q<h \\
& f_{I V}=\frac{32(h+k-q)^{5}}{35 h^{2} k^{2} q^{2}}\left(2 h^{4}+q\left(h^{3}+k^{3}\right)-h^{3} k+q^{2}\left(h^{2}+k^{2}\right)+h^{2} k^{2}-h k^{3}+q^{3}(h+k)+2 k^{4}+2 q^{4}\right), \begin{array}{l}
q>h \\
q>k
\end{array}
\end{aligned}
$$

Combining the expressions in Eqs. (17) - (20), we see that $f$ is a continuous and nonnegative function that neatly separates into five distinct domains:

$$
f(h, k, q)= \begin{cases}f_{I}, & 0<q \leq h, 0<q \leq k \\ f_{I I}, & h<q \leq k \\ f_{I I I}, & k<q \leq h \\ f_{I V}, & q>h, q>k, q<h+k \\ 0, & \text { otherwise }\end{cases}
$$

As illustrated in Figure 2, where the axes are normalized to the scattered photon energy $q$, the domains of $f$ are made up of three rectangular regions and two triangular regions. The function in rectangular regions II and III are mirror images - one can go from one to the other by interchanging $h$ and $k$. The triangular border of region IV is necessitated by energy conservation, as the energy of the scattered photons cannot exceed the total energy of the incident photons. Using this definition of $f$, the temporal evolution of an arbitrary photon distribution under photon-photon scattering may finally be expressed as:

$$
\frac{d n_{k}^{\prime}}{d t}=\frac{1}{(2 \pi)^{3}} \frac{139 e^{8}}{2025 m^{8}} \int h^{2} d h k^{2} d k f(h, k, q)\left[n_{h} n_{k}\left(1+n_{h}^{\prime}\right)\left(1+n_{k}^{\prime}\right)-n_{h}^{\prime} n_{k}^{\prime}\left(1+n_{h}\right)\left(1+n_{k}\right)\right]
$$

As a first example, we examine the behavior of a monochromatic but otherwise random photon gas. The first order response of this distribution is shown in Figure 3. An initial photon gas comprised of a single frequency $\omega_{0}$ will create a band of new frequencies extending from 0 to $2 \omega_{0}$ peaking at $\omega_{0}$. Similarly, when there are two different spectral energies in the photon gas, the newly scattered photons will tend to possess energy in between their parent photons, also shown in Figure 3. 
The mechanism for the creation of photons with different energies may be summarized as follows. Counter-propagating photons collide head-on and scatter back into photons at the initial frequency $\omega_{0}$. Co-propagating photons do not interact because they cannot interfere.

Interference is required because the scattering is mediated by the production and annihilation of a virtual electron-positron pair, which has non-zero rest mass. Interfering photons imply the existence of (non-propagating) electromagnetic energy with no net momentum, thus allowing for 4-momentum conservation between massless and massive particles. This is directly reflected by the Heisenberg-Euler interaction Lagrangian density [1],

$$
\begin{aligned}
& \mathcal{L}=\frac{1}{4 \pi \alpha}\left[\mathcal{F}+\frac{2 \alpha}{90 \pi}\left(4 \mathcal{F}^{2}+7 \mathcal{G}^{2}\right)\right] \\
& \mathcal{F}=\frac{1}{4} F_{\mu \nu} F^{\mu \nu}=\frac{1}{2}\left(\mathbf{B}^{2}-\mathbf{E}^{2}\right) \\
& \mathcal{G}=\frac{1}{4} F_{\mu \nu} F^{* \mu \nu}=\frac{1}{8} F_{\mu \nu} \varepsilon^{\mu \nu \sigma \lambda} F_{\sigma \lambda}=-\mathbf{B} \cdot \mathbf{E}
\end{aligned}
$$

which is constructed around the Lorentz-invariant quantities $\mathcal{F}$ and $\mathcal{G}$. These quantities are identically zero for co-propagating plane waves, reflecting the aforementioned 4-momentum conservation requirement. In equation (23), $\varepsilon^{\mu \nu \sigma \lambda}$ is the completely anti-symmetric Levi-Civita tensor [14]. One can also deduce this fact directly from the nonexistence of the CM frame when $\mathbf{h}$ and $\mathbf{k}$ are collinear.

All other situations produce photons that, when observed in the rest frame of the photon gas, possess energies different from $\omega_{0}$ and determined by geometry; finally 4-momentum conservation bounds the maximum photon energy after $n$ collisions to $n \omega_{0}$. As time marches forward, this new distribution of photons will create new photons while removing previously scattered photons, and in this way continuously modify the photon spectral distribution.

To illustrate the process, we will assume that an initial gas of photons can be described by Maxwell-Boltzmann distribution and analyze its temporal evolution toward equilibrium. By quantum kinetic theory, the equilibrium distribution is described by a Bose-Einstein distribution. The initial distribution is normalized such that the photon number density and energy density are 
identical to that of an ideal blackbody distribution at a given temperature $\xi=k_{B} T / m c^{2}$. Therefore, we have $n_{k}(t=0)=e^{-\alpha-\beta k}$ with $\alpha=\ln \left(\frac{\pi^{12}}{90^{3} \zeta(3)^{4}}\right)$ and $\beta=\frac{90 \zeta(3)}{\pi^{4} \xi}$. Here, $\zeta(z)$ denotes the Riemann zeta function. The photon number densities of the initial and equilibrium distributions are shown in Figure 4.

A numerical implementation of our photon evolution model was carried out in Mathematica using a simple time-stepping algorithm. The temporal evolution described by equation (22) can be well approximated by Euler's method,

$$
n_{k}(t+\Delta t) \approx n_{k}(t)+\frac{d n_{k}}{d t} \Delta t
$$

as long as $\Delta t$ is suitably small. At each time step, a new photon distribution is generated based on the differential scattering rate over a predefined nonuniform $k$-grid, which is then used to calculate the subsequent time step. More sophisticated computer algorithms exist which may further improve numerical accuracy and computation speed (c.f. Ref [15]). However, the rudimentary numerical scheme used here is sufficient in demonstrating the concept.

Figure 5 shows the spectrally-resolved rate of change of a photon gas with $\xi=0.01$ as it evolves in time. At large $t$, the rate of change tends to zero as the photon gas approaches equilibrium state. It is convenient to define a characteristic time $\tau(t)$ as:

$$
\frac{1}{\tau(t)}=\frac{\int\left|\frac{d n_{k}}{d t}\right| k^{2} d k}{\int n_{k} k^{2} d k} .
$$

For the initial Maxwell-Boltzmann distribution used in this calculation, direct numerical integration gives $\frac{1}{\tau_{0}} \approx \frac{1}{(2 \pi)^{3}} \frac{139 e^{8}}{2025 m^{8}} \xi^{9} \times 206.6$ at $t=0$. For $\xi=0.01$, the characteristic time $\tau_{0}$ is close to 8 million seconds, or 92 days. Due to the strong $\xi^{9}$ dependence, however, the 
characteristic time to reach equilibrium diminishes rapidly at higher temperatures $-\tau_{0}$ reduces to mere seconds at $\xi=0.05$. Figure 6 shows a linear time dependence for $\tau(t)$, suggestive of logarithmic approach towards equilibrium. The slight deviation at large $t$ is likely attributed to numerical artifacts due to the loss of grid resolution as the peak of the spectral change moves toward smaller values of $k$. Note that it has been implicitly assumed that the evolution timescale is much longer than $2 \pi / k$, so that it is meaningful to speak of time and instantaneous frequency simultaneously. Within the regime of validity of the Heisenberg-Euler theory, this assumption is generally very good.

Applications of the foregoing analysis may now be outlined for the Hawking-Unruh effect and early universe astrophysics. As far as the Hawking-Unruh effect is concerned, let us first recall that for an accelerated observer, the vacuum quantum fluctuations appear as thermal radiation, with a temperature proportional to the acceleration $a$ [5]:

$$
T=\frac{\hbar a}{2 \pi c k_{B}}
$$

This suggest that, for arbitrarily high acceleration, very high temperatures could be observed in that particular frame, leading to the question of whether nonlinear QED effect due to photonphoton scattering effects may be observed, and potentially violating general covariance principles by generating a different photon distribution. This does not occur, since we have shown that the equilibrium Bose-Einstein distribution of the photon gas is unperturbed by nonlinear photon-photon scattering under the conditions studied. In a more general context, the gedanken experiment discussed above points to the notion that spin, a key symmetry of the Lorentz group, is also fundamentally linked to general covariance.

The numerical model presented above for the transient evolution of a homogeneous, isotropic photon gas within the context of the Euler-Heisenberg nonlinear QED vacuum has been derived from fundamental scattering principles and the Boltzmann transport equation. Transient evolution from arbitrary initial photon distribution can be modeled using this formalism. Explicit calculations using a Maxwell-Boltzmann initial photon distribution are presented, 
clearly showing temporal evolution towards Bose-Einstein equilibrium. Results confirm the evolution of the radiation content of the Universe, as described in the standard Lambda-CDM model since, even at high temperatures and under nonlinear QED (and other) processes, Pauli's spin-statistics relation remains valid, and photons maintain the Bose-Einstein distribution in equilibrium. Concepts discussed here though simplistic may have far-reaching implications for astrophysics and cosmology as well as high energy density (HED) physics. The authors hope that this basic study will provide insights to fundamental questions and engender continuing interest and discussion on this subject.

This work performed under the auspices of the U.S. Department of Energy by Lawrence Livermore National Laboratory under Contract DE-AC52-07NA27344. 
Figures:

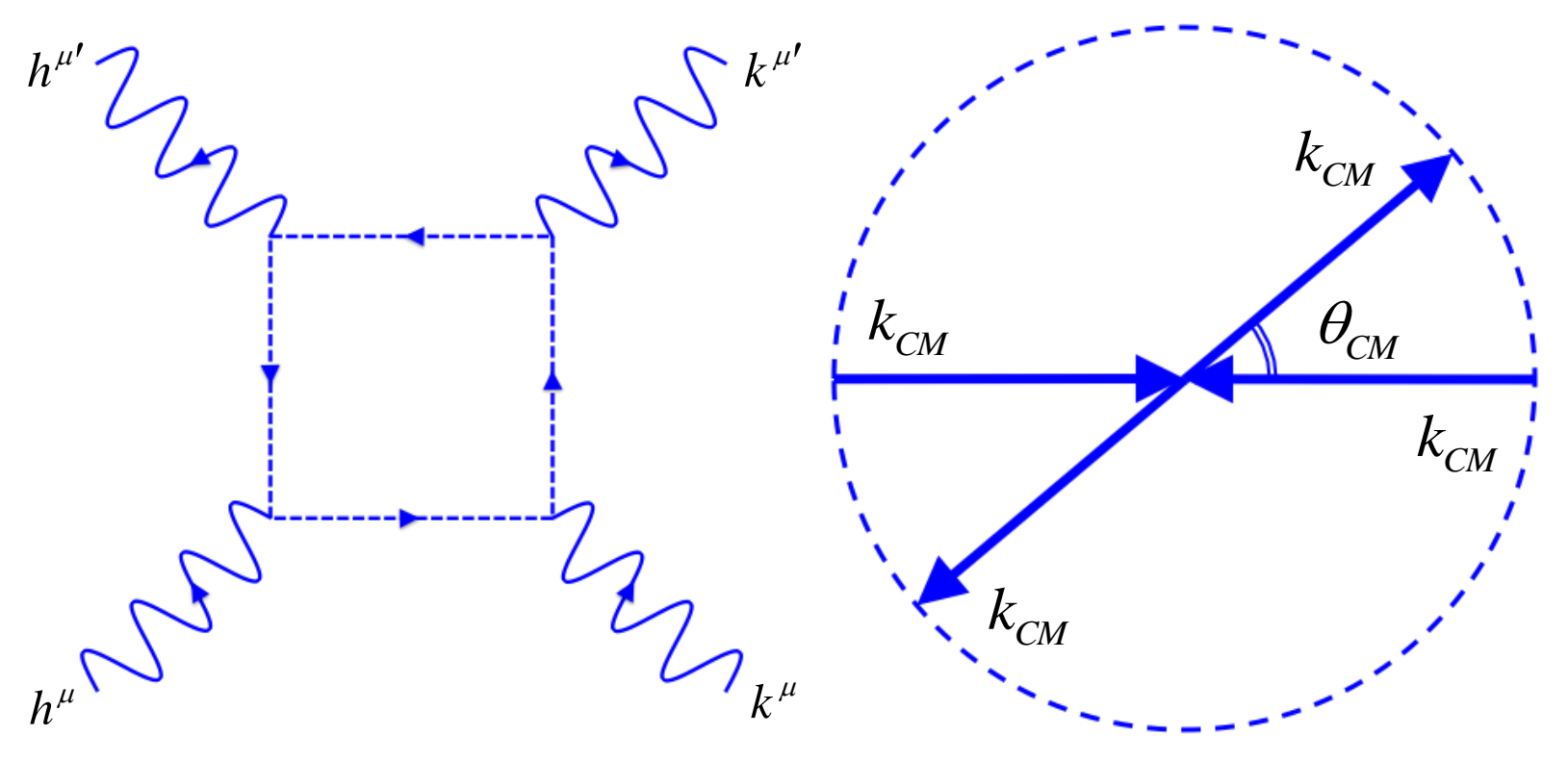

Figure 1. Left: Feynman diagram for lowest order photon-photon scattering. Right: Photon-photon interaction geometry in the center of mass (CM) frame. $h$ and $k$ represent incident photons and $h$ ' and $k$ ' represent the scattered photons. In the $\mathrm{CM}$ frame, all interacting photons possess the same energy. 


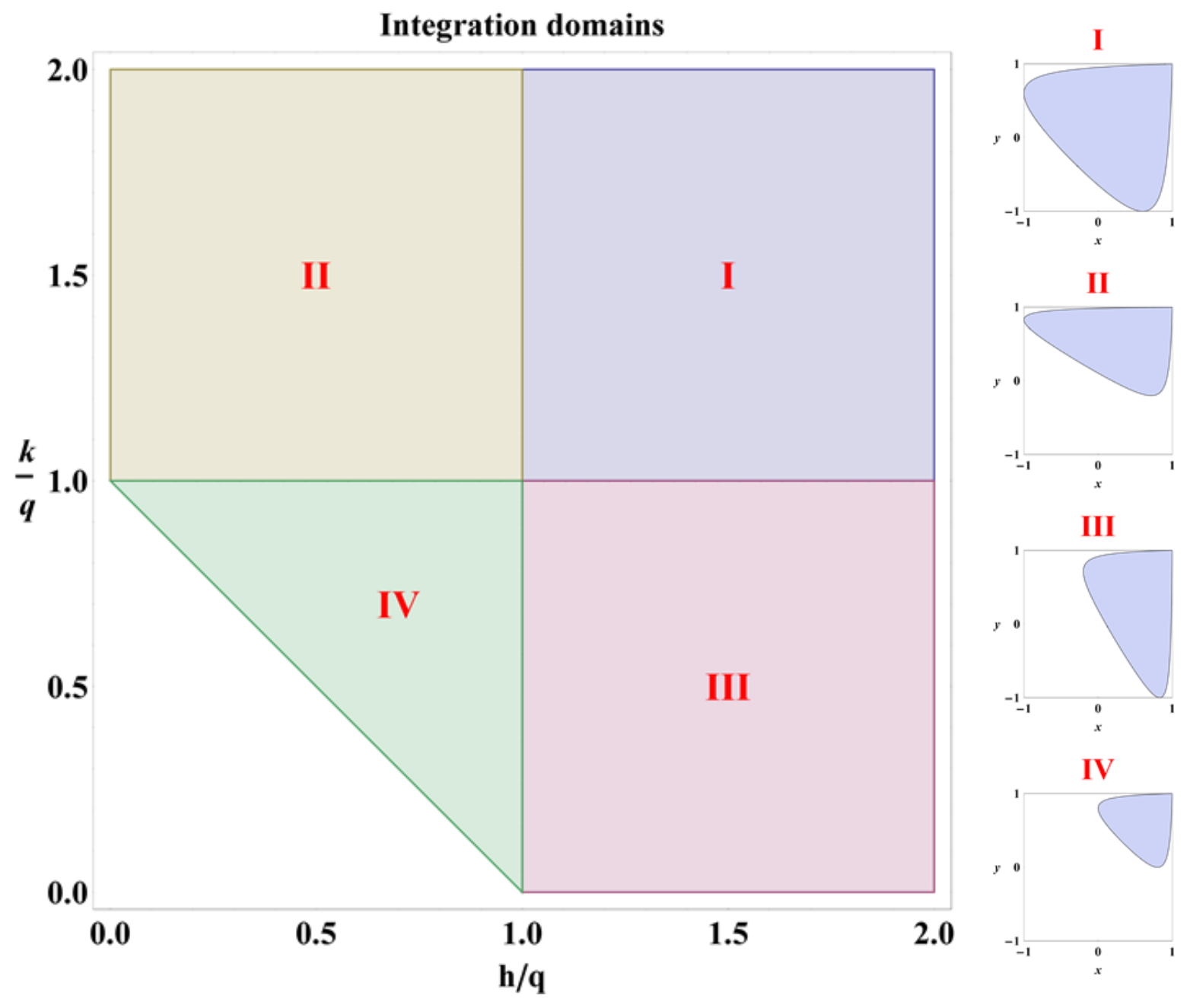

Figure 2. Shown are the integration domains used in the calculations for the collision kernel $f$ based on the values of $h, k$ and $q$ (left) and their corresponding integration regions in $x-y$ space (right). The triangular border of region IV is necessitated by energy conservation, as the energy of the scattered photons cannot exceed the total energy of the incident photons. 


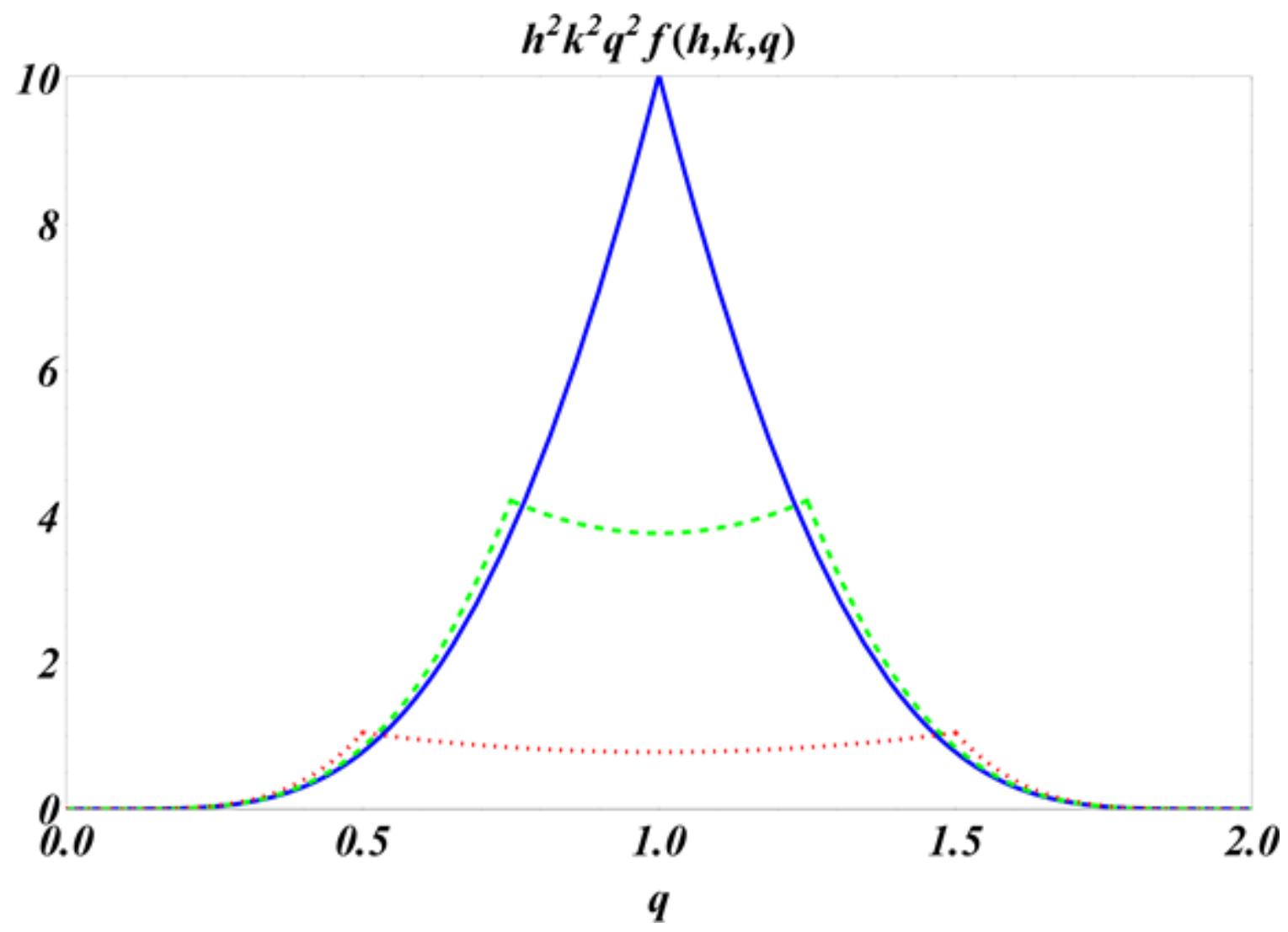

Figure 3. Plot of the collision kernel $f(h, k, q)$ as a function of the scattered energy $q$ with different energy values for the incident photons: (a) $h=k=1$ (solid); (b) $h=3 / 4, k=5 / 4$ (dashed); (c) $h=1 / 2, k=3 / 2$ (dotted). 


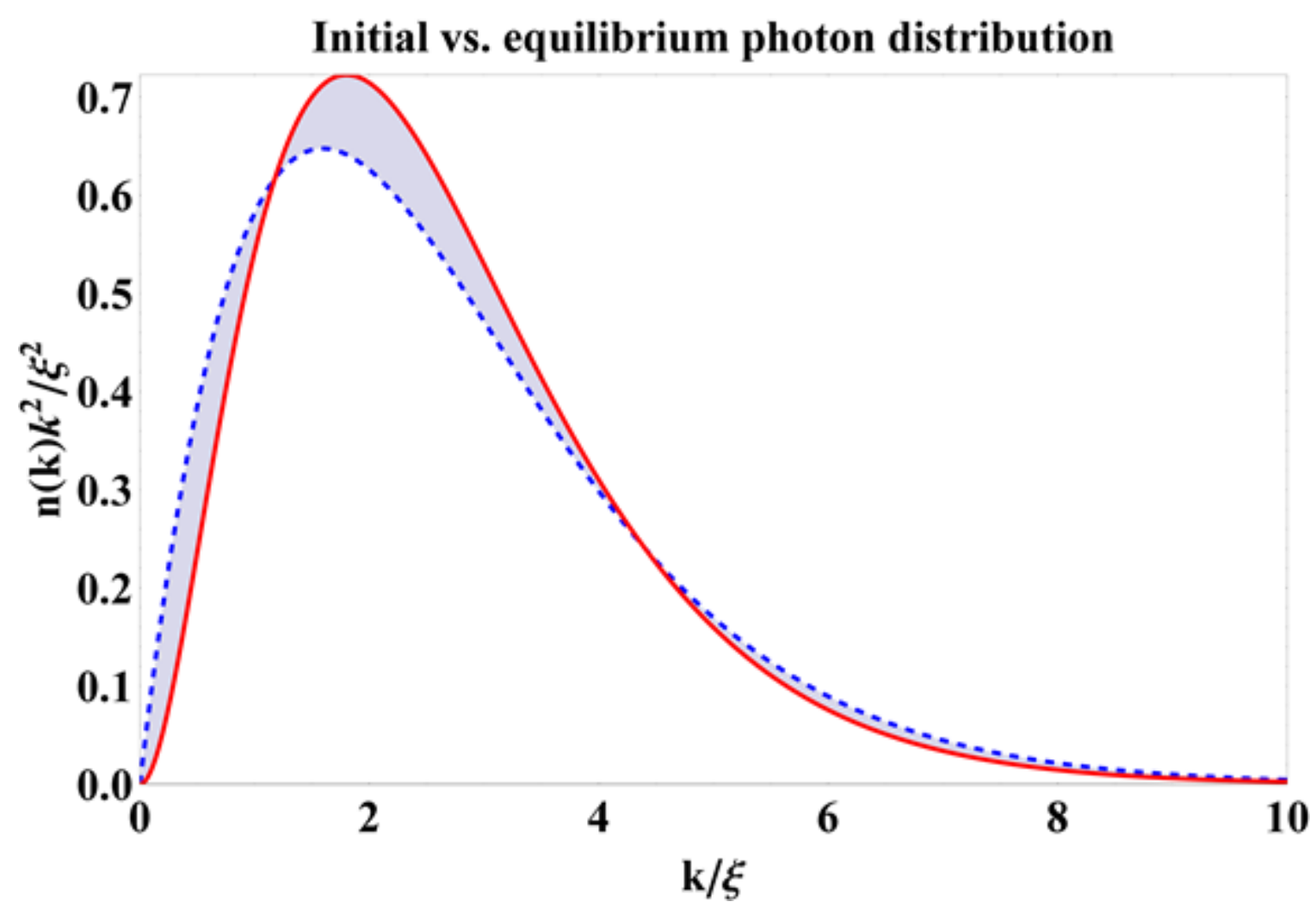

Figure 4. Normalized Maxwell-Boltzmann initial photon distribution satisfying (dashed) versus the Bose-Einstein equilibrium distribution (solid). Photon energy $k$ is measured relative to the electron rest energy. $\xi$ is a predetermined temperature parameter defined as $\xi=k_{B} T / m c^{2}$.

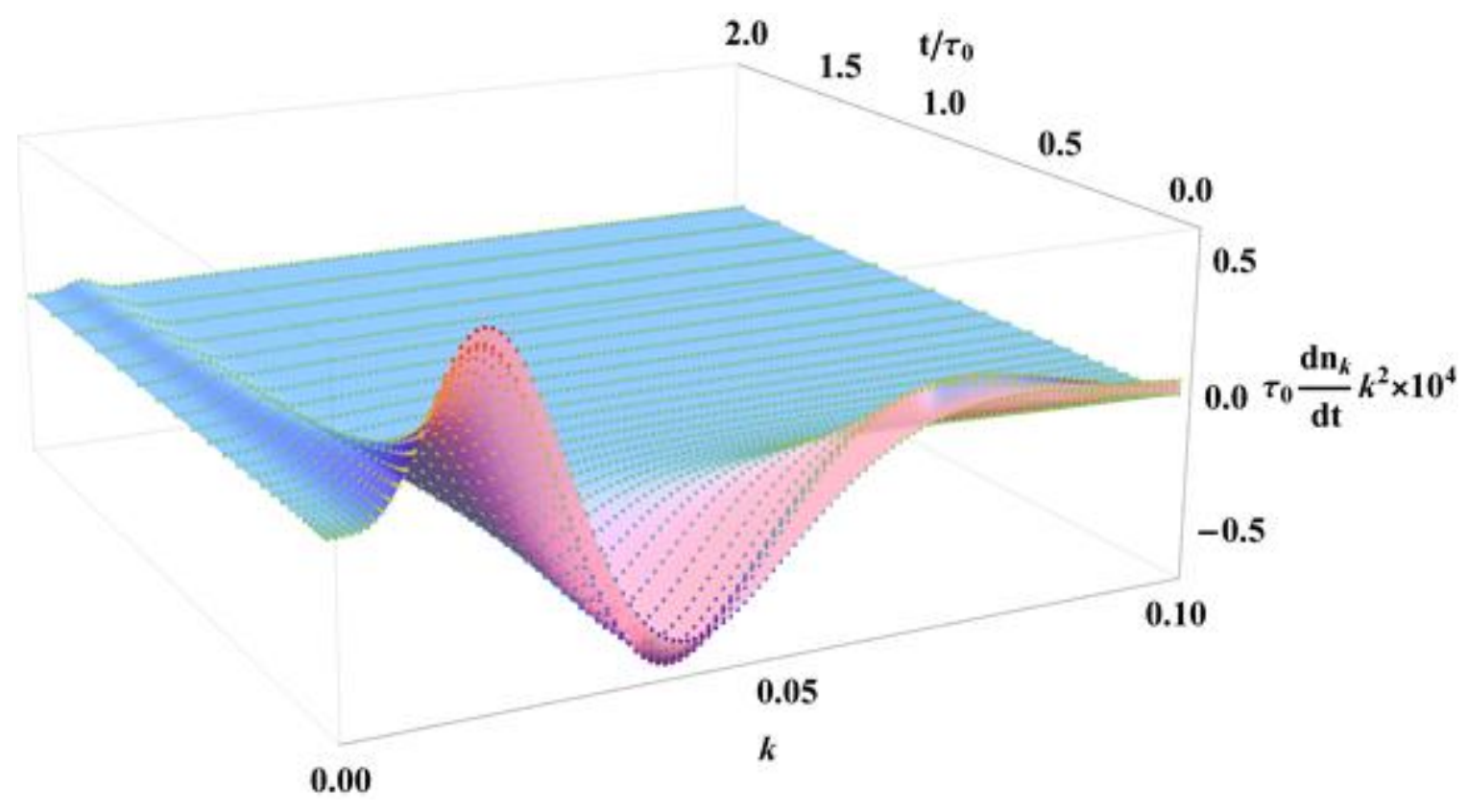

Figure 5. The differential rate of change of the photon distribution over time for $\xi=0.01$. The characteristic time scale $\tau_{0}$ is defined by equation (25) evaluated at $\mathrm{t}=0$. Photon energy $k$ is in fractions of the electron rest energy. 


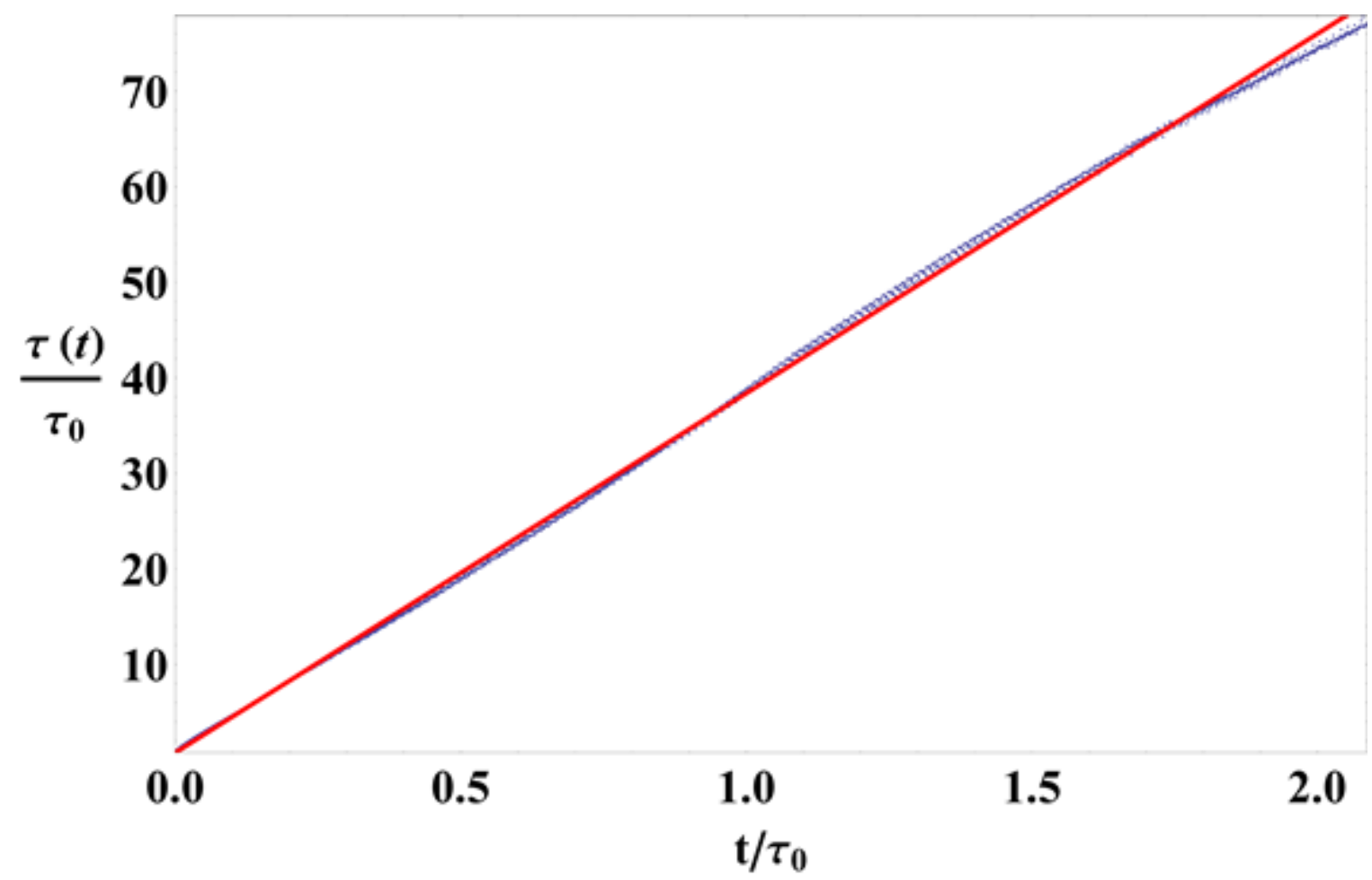

Figure 6. This is a plot of the characteristic time $\tau(t)$ defined in equation (25) using model results to show steady increase during transient evolution. $\tau_{0}$ denotes the initial characteristic time evaluated at $\mathbf{t}=\mathbf{0}$. The solid line is a linear fit to the numerical data. 


\section{References}

[1] W. Heisenberg, H. Euler, "Folgerungen aus der Diracschen Theorie des Positrons", Zeitschr. Phys. 98, 714 (1936).

[2] M. Marklund, P.K. Shukla, "Nonlinear collective effects in photon-photon and photon-plasma interactions", Rev. Mod. Phys. 78, 591-640 (2006)

[3] G.F. Smoot, "Nobel Lecture: Cosmic microwave background radiation anisotropies: Their discovery and utilization", Rev. Mod. Phys. 79, 1349-1379 (2007)

[4] M. Bartelmann, "The dark Universe", Rev. Mod. Phys. 82, 331-382 (2010)

[5] W.G. Unruh, "Notes on black-hole evaporation", Physical Review D 14 (4): 870

[6] V.B. Berestetskii, E.M. Lifshitz, L.P. Pitaevskii, Quantum Electrodynamics. Vol. 4 (2nd ed.), ButterworthHeinemann (1982).

[7] L.P. Pitaevskii, E.M. Lifshitz, Physical Kinetics. Vol. 10 (1st ed.), Pergamon Press (1981).

[8] H. Smith, H.H. Jensen, Transport Phenomena, Oxford University Press, New York (1989)

[9] T. Padmanabhan, Theoretical Astrophysics, Vol 1: Astrophyical Processes, Cambridge University Press (2000)

[10] R.A. Sunyaev, Ya.B. Zel'dovich, "Microwave background radiation as a probe of the contemporary structure and history of the universe", Ann. Rev. Astro. Astrophys 18: 537-560 (1980).

[11] A.S. Kompaneets, "The Establishment of Thermal Equilibrium between Quanta and Electrons", Sov. Phys. JETP, 4, 730 (1957).

[12] J.R. Buchler, W.R. Yueh, The Astrophysical Journal, 210: 440-446 (1976)

[13] Wolfram Research, Inc., Mathematica, Version 8.0, Champaign, IL (2010).

[14] T. Levi-Civita, The Absolute Differential Calculus (Calculus of Tensors), Dover, New York (1977)

[15] P. A. Markowich, L. Pareschi. "Fast conservative and entropic numerical methods for the Boson Boltzmann equation”, Numer. Math. 99, 3 (January 2005), 509-532. 